\title{
1 Precise measurement of selenium isotopes by HG-MC-ICPMS using a 76-78 double-spike
}

2 Marie-Laure Pons ${ }^{\mathrm{a}, \mathrm{b}^{*}}$, Marc-Alban Millet ${ }^{\mathrm{c}}$, Geoff N. Nowell ${ }^{\mathrm{d}}$, Sambuddha Misra ${ }^{\mathrm{a}, \mathrm{e}}$ and Helen

M. Williams ${ }^{\mathrm{a} \dagger}$

4

5 a The University of Cambridge, Department of Earth Sciences, Downing St, Cambridge CB2

6 3EQ, United Kingdom

$7 \quad{ }^{\mathrm{b}}$ CNRS, Aix Marseille Univ, IRD, INRA, Coll France, CEREGE, 13545, Aix en Provence,

8 France

9

${ }^{\mathrm{c} C a r d i f f}$ University, School of Earth and Ocean Sciences, Main Building, Park Pl, Cardiff CF10 3AT, United Kingdom

${ }^{\mathrm{d}}$ Durham University, Department of Earth Sciences, Elvet Hill, Durham DH1 3LE, United Kingdom

${ }^{\text {e}}$ Indian Institute of Science, Centre for Earth Sciences, Bengaluru, India

*corresponding author dr.marie.laure.pons@gmail.com

\section{Abstract}

Selenium (Se) stable isotopes are a new geochemical tool that with great potential as a tracer of redox processes and the chemical cycling of chalcophile and volatile elements. However, Se isotope measurements in low-Se samples present a formidable analytical challenge. In this study, we report a new method to measure Se stable isotopes $\left(\delta^{82 / 78} \mathrm{Se}\right.$; per mil deviation relative to Se NIST SRM 3149) at extremely high precision. Selenium has six stable isotopes and therefore is a good candidate for isotope analysis using a double spike approach, which has the advantage that it can correct for any stable isotope fractionation that may occur during sample

\footnotetext{
${ }^{\dagger}$ Electronic supplementary information (ESI) available.
} 
processing. We have calibrated a novel ${ }^{76} \mathrm{Se}-{ }^{78} \mathrm{Se}$ double spike and have developed a rapid and precise analytical protocol on a multi-collector inductively coupled plasma mass spectrometer using an ESI hydride generation introduction system. Sensitivity is over 1000V/ppm for total Se signal; a measurement typically requires $25 \mathrm{ng}$ of natural Se. Argon dimer interferences on masses 76,78 and 80 were corrected in-situ by measuring mass 80 . Germanium interferences on masses 74 and 76 were corrected by measuring mass 73 and mass 75 was monitored to correct for arsenic hydride on mass 76 . Wash-out times were in the order of $180 \mathrm{~s}$, greatly reduced compared to previous studies that rely on an on peak zero argon dimer corrections (wash-out times up to one hour). The 2 s.e. error for a single analysis typically ranges from 0.01 to $0.025 \%$ (n=80) for $\delta^{82 / 78} \mathrm{Se}$. Our long-term reproducibility and accuracy were estimated by multiple analyses of the Se Merck standard over numerous different analytical sessions, resulting in a mean $\delta^{82 / 78} \mathrm{Se}$ value of $-0.989 \pm 0.034 \%$ ( $\mathrm{n}=93 ; 2$ s.d.), which is in excellent agreement with previous studies.

\section{Introduction}

Recent progress in plasma source multi-collector mass spectrometry in the last decades have enabled the development of the techniques for the measurement of previously unexplored isotope systems, the so-called "non-traditional" elements, including magnesium, iron, titanium, molybdenum, chromium and selenium ${ }^{1-6}$. Selenium $(\mathrm{Se})$ has six stable isotopes $\left({ }^{74} \mathrm{Se}, 0.889 \%\right.$;

${ }^{76} \mathrm{Se}, 9.366 \% ;{ }^{77} \mathrm{Se}, 7.635 \% ;{ }^{78} \mathrm{Se}, 23.772 \% ;{ }^{80} \mathrm{Se}, 49.607 \%$ and ${ }^{82} \mathrm{Se}, 8.731 \%$ ), displays similar chemical behaviour to sulphur, and exhibits multiple redox states, from reduced $\mathrm{Se}(-\mathrm{II})$ to most oxidized $\mathrm{Se}(\mathrm{VI})$. For these reasons, Se stable isotopes have received considerable attention from the geochemistry community. For example, previous studies have demonstrated that $\mathrm{Se}$ stable isotopes have the potential to constrain the redox evolution of the Earth's ocean and atmosphere through time using sulphide and Se-rich sedimentary records ${ }^{7-14}$, and Se isotopes 
have also been used to constrain redox processes and biogeochemical cycling in the critical zone $^{15-20}$.

However, the Se isotope compositions of the Earth's mantle and other major chemical reservoirs remain poorly constrained ${ }^{21-25}$, limiting the further application of this system to terrestrial and planetary geology. This is in part due to the very low abundances of Se in mantle rocks (typically 3-100 $\mathrm{ppb}^{26-28}$ ) coupled with multiple analytical challenges, such as the low ionisation efficiency of Se in argon plasmas as well as multiple interferences from argide species as well as germanium and arsenic, which are present at significant concentrations in target samples. A number of different analytical strategies have been employed to deal with these challenges, all of which have their own inherent advantages and disadvantages ${ }^{6,29-35}$. Here we present a method allowing measurements of Se stable isotopes $\left(\delta^{82 / 78} \mathrm{Se}\right.$; per mil deviation relative to Se NIST SRM 3149) in geological samples at increased precision. We have employed a double spike approach, as previous studies have demonstrated the potential of this method to yield highly accurate and precise stable isotope ratio measurements ${ }^{2,36-40}$ and focussed on the development of a new method to measure Se stable isotopes to high precision.

\section{Double spike}

Selenium stable isotope measurements are fraught with difficulty. In addition to isobaric argonbased interferences on masses 74, 76, 78 and 80, non-quantitative yields during Se purification by ion exchange chromatography as well as the large degree of instrumental mass bias that is inherent to plasma mass spectrometry can cause reduced precision and/or inaccuracy if not accounted for properly. The double spike method is ideally suited to generate highly precise stable isotope measurements for elements with 4 or more isotopes as it can reliably account for laboratory based stable isotope fractionation (i.e. the combined effects of sample processing 
and mass spectrometry) and also provides an exceptionally precise means of correcting for instrumental mass bias ${ }^{36-42}$. As such it has been widely used by isotope geochemists in the development of high-precision analytical methods for a wide range of non-traditional stable isotope systems over the last decade ${ }^{2,3,43-48}$. The double spike method relies on the addition of an isotope tracer (or spike) of known composition to the sample of interest during sample processing. The final error on the stable isotope ratio is strongly dependent on the isotope composition of the spike as well as the mixing proportion between sample and spike. Typically, spikes made out of the mixture of two isotope only (i.e. 'double' spikes) have proven favourable over spikes made of three isotopes (i.e. 'triple' spikes ${ }^{48}$ ), providing the composition of the 'double spike' is chosen carefully. In order to do this we modelled optimum spike compositions and spike-sample proportions for Se stable isotope measurements using the approach outlined by Millet and Dauphas in $2014^{2}$, which takes into account the errors associated with counting statistics and collector Johnson noise (see ref. 2 for more details). Johnson noise, i.e. the thermal noise generated in the Faraday cup collectors, although low at room temperatures and negligible compared to counting statistics for ion beams over a few hundred millivolts, can nonetheless add significant amount of uncertainty when low ion beams are measured. In our models we assumed beam conditions were set as $35 \mathrm{~V}$ for the most abundant isotope of each mixture and all ion beams collected in collectors with $10^{11} \Omega$ amplifiers apart from ${ }^{80} \mathrm{Se}$, where a $10^{10} \Omega$ amplifier was employed. Room temperature was set to $25^{\circ} \mathrm{C}$ and measurements consisted of 80 integrations $4.194 \mathrm{~s}$ each.

Using our model approach, two potential double spikes were found to provide low errors, an ${ }^{82} \mathrm{Se}-{ }^{78} \mathrm{Se}$ spike similar to that used by Pogge et al. $(2014){ }^{33}$ and $\mathrm{a}^{76} \mathrm{Se}-{ }^{78} \mathrm{Se}$ double spike (Fig. 1) that allows slightly better internal precision. Both of these potential double spike solutions use ${ }^{76} \mathrm{Se},{ }^{77} \mathrm{Se},{ }^{78} \mathrm{Se}$ and ${ }^{82} \mathrm{Se}$ isotope signals for the spike deconvolution ${ }^{33}$. The ${ }^{76} \mathrm{Se}-{ }^{78} \mathrm{Se}$ spike provides the advantage of being able to generate low analytical uncertainties over a much larger 
range of spike-sample mixing ratios (Fig. 1). This is particularly important in the case of lowabundance elements like Se where element concentrations may not be known at high precision prior to isotope measurements. However, a potential drawback in using this spike is that there are significant argide isobaric interferences on both ${ }^{76} \mathrm{Se}$ and ${ }^{78} \mathrm{Se}\left({ }^{36} \mathrm{Ar}^{40} \mathrm{Ar}\right.$ and ${ }^{38} \mathrm{Ar}^{38} \mathrm{Ar}$ on mass $76 ;{ }^{38} \mathrm{Ar}^{40} \mathrm{Ar}$ on mass 78 , respectively) in contrast to the previously used selenium double spikes such as ${ }^{74} \mathrm{Se}-{ }^{77} \mathrm{Se}$ and ${ }^{78} \mathrm{Se}-{ }^{82} \mathrm{Se}$, which only have one interfered isotope (minor ${ }^{36} \mathrm{Ar}{ }^{38} \mathrm{Ar}$ interference on mass 74 and significant ${ }^{38} \mathrm{Ar}^{40} \mathrm{Ar}$ interference on mass 78 , respectively $\left.{ }^{6,29,33,34}\right)$.

The presence of these argon dimer interferences requires precise interference corrections prior to spike deconvolution. In addition to argides, ${ }^{76} \mathrm{Ge}$ and ${ }^{75} \mathrm{AsH}$ can also interfere on mass 76 , making the ${ }^{76} \mathrm{Se}$ signal challenging to decipher. However, as detailed below, we can mitigate against the effects of these interferences by developing a new protocol that allows for better correction of argon-based interferences as well as efficient arsenic hydride and germanium interferences correction on mass 76 . For these reasons, we were able to take advantage of the additional precision that a ${ }^{76} \mathrm{Se}-{ }^{78} \mathrm{Se}$ double spike can offer, with an optimal DS-sample mixture of 50:50. Furthermore, the use of a ${ }^{76} \mathrm{Se}-{ }^{78} \mathrm{Se}$ double spike can be advantageous in certain circumstances as it serves to boost the ${ }^{76} \mathrm{Se}$ signal (also a critical isotope in ${ }^{82} \mathrm{Se}^{78} \mathrm{Se}$ double spike studies ${ }^{33}$ ) and therefore decrease the proportion of interfering species on a mass of interest.

\section{Experimental}

\subsection{Samples and reagents}

All of the sample preparation, including the double spike mixing, were carried out in the ultraclean part of the Arthur Holmes clean chemistry laboratory (class 1000 clean room) at Durham University, in a bespoke class 100 extraction cabinet. 
Selenium spikes were purchased from Isoflex USA, both in elemental form. The ${ }^{76}$ Se spike has an enrichment level of $99.80 \pm 0.10 \%$ (certificate of analysis number 3598 ). The ${ }^{78}$ Se spike has an enrichment level of $99.30 \pm 0.10 \%$ (certificate of analysis number 3009 ). The ${ }^{76} \mathrm{Se}$ and ${ }^{78}$ Se spikes were mixed in a new and cleaned PTFE Teflon bottle.

Standards

In this study, two selenium mono-elemental solutions with different Se isotopic compositions were used. Results were reported against a National Institute of Standards and Technology pure Se standard, NIST SRM-3149 (lot number 200901, $10 \mathrm{~m} \cdot \mathrm{g}^{-1}$ ). This single element standard solution, while not isotopically certified, is now widely used as the reference material for Se isotope measurements ${ }^{13,21,33,35,49,50}$ and its absolute composition has been reported ${ }^{51}$. The second selenium standard solution used in our study is a MERCK pure Se solution which was already used as reference by Rouxel and collaborators in their pioneering study in 2002 and was further characterised against NIST SRM-3149 by Carignan and Wen in 20076,35. For the germanium and arsenic doping experiments, mono-elemental Ge and As Specpure 1000 ppm Alpha Aesar solution were used.

\section{Reagents}

Distilled $\mathrm{HCl}$ was used to dilute the samples for Se isotope measurements and to generate hydrides (see 3.2.). Two other reagents were used in the hydride generation reaction (see Eq. 1): analytical grade sodium borohydride and extra pure sodium hydroxide pellets from Fischer Chemical. 


\section{Hydride generation}

Selenium stable isotope measurements were performed with a Thermo Scientific ${ }^{\mathrm{TM}}$ Neptune Plus $^{\mathrm{TM}}$ multi-collector ICP-MS coupled to an ESI HydrideICP hydride generation introduction system. We employed a hydride generator because numerous previous studies ${ }^{29,33,34,52}$ have shown that Se ionises far more efficiently as a hydride, rather than in elemental form. However, it should be noted that we measured Se isotopes on-mass as opposed to measuring the masses of Se-hydride molecular ions (which presumably break down in the plasma itself, before ionisation). The ESI hydride generator system features a micro peripump that leads the reagents and sample through a PFA mixing block to a low-volume quartz gas-liquid separator where hydrides are formed and separated from liquid waste then carried via argon sample gas to the plasma torch via an intermediary ESI Stable Sample Introduction dual quartz chamber, which was used to stabilise the signal. The hydrides are formed by reacting the sample, conditioned in $1 \mathrm{M} \mathrm{HCl}$, with sodium borohydride (1 wt $\% \mathrm{NaBH}_{4}$ in $\left.0.012 \mathrm{M} \mathrm{NaOH}\right)$ and reducing $\mathrm{Se}(\mathrm{IV})$ to $\mathrm{Se}(-\mathrm{II})^{33}$. Prior to analysis, the sample is oxidized and conditioned in $1 \mathrm{M} \mathrm{HCl}$. The ESI HydrideICP has three uptake lines: one for the sample, one for $\mathrm{NaBH}_{4}$ and one for $1 \mathrm{M} \mathrm{HCl}$ and solution uptake rates were adjusted on a daily basis (see Table 1). The sodium borohydride was prepared on the day of the analysis as it is known to degrade by $\mathrm{H}_{2}$ production ${ }^{33}$. The hydride generation reaction is below:

$$
3 \mathrm{NaBH}_{4}+3 \mathrm{H}_{2} \mathrm{O}+3 \mathrm{HCl}+2 \mathrm{H}_{2} \mathrm{SeO}_{3} \leftrightarrow 3 \mathrm{NaCl}+2 \mathrm{H}_{2} \mathrm{Se}+3 \mathrm{H}_{3} \mathrm{BO}_{3}+6 \mathrm{H}_{2} \quad \text { Eq. } 1
$$

\section{MC-ICPMS operating parameters and analytical protocol}

Selenium stable isotope measurements were carried out on a Thermo Fisher Scientific ${ }^{\text {TM }}$ Neptune Plus ${ }^{\mathrm{TM}}$ MC-ICPMS at the Earth Sciences Department of the University of Durham, 
UK. Samples were introduced through an ESI Hydride generator and an ESI Stable Sample Introduction dual quartz chamber using argon as a carrier gas. We used regular nickel sample and skimmer cones and the analysis were carried out in low resolution mode (see Table 3 operating parameters). The isotope ratio measurements consisted of one block of 80 integrations of 4.194 seconds each. The central cup was set on mass 77 and mass 80 was measured on the High-2 cup using a $10^{10} \Omega$ amplifier that can accommodates a signal of up to $500 \mathrm{~V}$ on $\mathrm{H} 2$. Samples and standards were measured in $1 \mathrm{M} \mathrm{HCl}$ after 80 seconds uptake time and the washouts were carried in $1 \mathrm{M} \mathrm{HCl}$ for 180 seconds. At the end of the washout period and before any sample or standard measurement - an on peak zero (OPZ) of 1 block (10 integrations of 4.194 seconds each) was performed in clean 1M HCl. All samples were bracketed with measurements of standards that were spiked and treated similarly to the samples, with concentrations and spike Se:natural Se concentration ratios carefully matched in order to account for variation in the intensity of residual interferences. The overall $\mathrm{Se}$ sensitivity in low resolution mode using the ESI HydrideICP is $1000 \mathrm{~V}$ per ppm (total Se beam) on average. All the data reported in this paper - unless stated otherwise - were obtained for a sample and standard concentration of $50 \mathrm{ppb}$, which gives a signal of $\sim 2-2.4 \mathrm{~V}$ on mass 82 . The multiple interference corrections used in this study are described below. All measurements are reported in the $\delta$ notation as relative per mil difference to the international Se standard NIST SRM 3149 as follows:

$$
\delta^{82 / 78} \mathrm{Se}_{\text {sample }}=\left[\left({ }^{82 / 78} \mathrm{Se}_{\text {sample }} /{ }^{82 / 78} \mathrm{Se}_{\mathrm{NIST}-\mathrm{SRM}-3149)-1}{ }^{*} 1000\right.\right.
$$

Eq. 2

Critically, the use of a double spike does not preclude the presentation of data in terms of stable isotope ratios employing a "spiked" isotope as the entire premise of using a double spike relies 
on mass dependent fractionation as applied to all measured isotopes used in the double spike deconvolution $^{3,48}$.

\subsection{Interferences correction}

The measurements of Se isotope ratios represents an enormous technical challenge, as all of the selenium isotopes present isobaric interferences from other elements (e.g. germanium) as well as polyatomic interferences from molecular compounds (e.g. argon dimers, Se hydrides). These interferences must be corrected prior to double-spike deconvolution, as shown in the isobaric interference correction flowchart (Fig. 2). The interferences on Se isotopes are also summarized in Table 2, along with the correction method used in this study, for each of them.

\section{Argon dimer corrections}

Argon dimer isobaric interferences constitute a technical challenge for the measurement of selenium isotopic ratios by plasma source mass spectrometry. Depending on the argon isotopes involved, argon dimers interfere with selenium isotopes at $\mathrm{m} / \mathrm{z}$ ratios of $74\left({ }^{38} \mathrm{Ar}^{36} \mathrm{Ar}^{+}\right), 76$ $\left({ }^{38} \mathrm{Ar}^{38} \mathrm{Ar}^{+},{ }^{40} \mathrm{Ar}^{36} \mathrm{Ar}^{+}\right), 78\left({ }^{40} \mathrm{Ar}^{38} \mathrm{Ar}^{+}\right)$and, mostly, $80\left({ }^{40} \mathrm{Ar}^{40} \mathrm{Ar}^{+}\right)$. The argide production depends on plasma energy fluctuation as well as on the mass spectrometer tuning parameters. For a Se isotopes measurement at $50 \mathrm{ppb} \mathrm{Se}$, with the operating parameters as described as in Table 1 , the contribution of ${ }^{40} \mathrm{Ar}^{40} \mathrm{Ar}^{+}$dimers at $\mathrm{m} / \mathrm{z}=80$ is typically of $\sim 13$ to $20 \mathrm{~V}$ on $\mathrm{H} 2$, while the contribution of ${ }^{80} \mathrm{Se}^{+}$is typically of $\sim 25 \mathrm{~V}$ on $\mathrm{H} 2$. Due to plasma instability, we found that using a background on-peak zero (OPZ) correction to correct for ArAr interferences on masses 76, 78 and 80, used in the double spike deconvolution generated significant errors of up to $1 \%$ on the $\delta^{82 / 78} \mathrm{Se}$ of the samples. Consequently, we developed an alternative approach to correct argon dimers at every integration during the measurement, broadly following the methods of Elwaer and Hintelmann $(2008)^{53}$ and Stuecken et al., $2013^{34}$, which account for 
plasma instabilities during the run. Given the IUPAC recommended values for the natural abundances of ${ }^{82} \mathrm{Se}$ and ${ }^{80} \mathrm{Se}^{54}$, we used the ${ }^{82} \mathrm{Se}$ beam to predict the contribution of ${ }^{80} \mathrm{Se}$ on the signal measured at $\mathrm{m} / \mathrm{z}=80$ (Fig. 2, purple path). In our method, there are no Se isotopes without background interferences, but among them, ${ }^{82} \mathrm{Se}$ requires the least interference correction, hence its use in our Ar dimer correction. The contribution of ${ }^{80} \mathrm{Se}$ at $\mathrm{m} / \mathrm{z}=80$ is thus:

$$
{ }^{80} S e={ }^{82} S e_{\text {corr }} * 49.80 / 8.82
$$

With ${ }^{82} \mathrm{Se}_{\text {corr }}$ being ${ }^{82} \mathrm{Se}$ corrected for ${ }^{82} \mathrm{Kr}$ using an on-peak zero subtraction for background Kr. During the OPZ performed at the end of the washout, ${ }^{83} \mathrm{Kr}$ is measured on mass 83 . Using $\mathrm{Kr}$ natural abundances ${ }^{54}$, the ${ }^{82} \mathrm{Kr}$ signal in the $\mathrm{OPZ}$ is calculated and this value in subtracted from the 82 beam during sample or standard measurements. The potential gas-based, bromine hydride interference ${ }^{81} \mathrm{BrH}$ on mass 82 is taken into account through sample standard bracketing.

The contribution of ${ }^{40} \mathrm{Ar}^{40} \mathrm{Ar}$ at $\mathrm{m} / \mathrm{z}=80$ is then:

$$
{ }^{40} A r^{40} A r=I(80)-{ }^{80} S e-{ }^{80} K r
$$

Eq. 4

With $\mathrm{I}(80)$ the measured intensity at $\mathrm{m} / \mathrm{z}=80$ and ${ }^{80} \mathrm{Kr}$ calculated using the $\mathrm{OPZ}{ }^{83} \mathrm{Kr}$ and $\mathrm{Kr}$ natural abundances. The potential gas-based, bromine hydride interference ${ }^{79} \mathrm{BrH}$ on mass 81 is taken into account through sample standard bracketing. The potential polyatomic ${ }^{40} \mathrm{Ar}^{40} \mathrm{Ca}$ interference on mass 80 is deemed negligible, as is no $\mathrm{Ca}$ in the pure Se standards used in this study, and for natural samples, the Se chemistry processing should remove any calcium ${ }^{34,49}$. 
249 The amount of ${ }^{40} \mathrm{Ar}^{40} \mathrm{Ar}$ is determined throughout the run for each integration. The contributions of other argon dimers at mass $76\left({ }^{36} \mathrm{Ar}^{40} \mathrm{Ar} ;{ }^{38} \mathrm{Ar}{ }^{38} \mathrm{Ar}\right)$ and $78\left({ }^{38} \mathrm{Ar}{ }^{40} \mathrm{Ar}\right)$ are deduced from the ${ }^{40} \mathrm{Ar}^{40} \mathrm{Ar}$ signal using the natural abundances of 76 and 78 argon dimers 0.006636 and 0.001257 respectively ${ }^{34,55}$. These contributions are then subtracted to the intensities at mass 76 and 78 (Fig. 2, purple path, argide correction arrows). This in-situ argide interference corrections greatly reduces the need for long on-peak zero (OPZ, or baseline) measurements and extended wash-out times (of up to one hour ${ }^{33}$ ), improving sample throughput and reducing reagent consumption.

\section{Germanium correction}

Naturally present Ge present in samples and incompletely separated from Se in column chemistry can also form hydrides $\left(\mathrm{GeH}_{4}\right)$ during the hydride generation process. Germanium possesses 5 stable isotopes $-{ }^{70} \mathrm{Ge},{ }^{72} \mathrm{Ge},{ }^{73} \mathrm{Ge},{ }^{74} \mathrm{Ge}$ and ${ }^{76} \mathrm{Ge}$, two of which $-{ }^{74} \mathrm{Ge}$ and ${ }^{74} \mathrm{Ge}-$ create isobaric interferences on mass 74 and mass 76 for the measurement of Se isotopes. Germanium corrections were performed using beam intensity at mass 73 (see Table 2 and Fig. 2). As the cup configuration we used for Se isotopes determination does not allow the simultaneous measurements of multiple germanium isotopes, mass bias correction could not be carried out independently for Ge and Se isotopes. Consequently, Ge and Se were assumed to have similar instrumental mass bias and a single instrumental fractionation factor $(\beta)$ was used in conjunction with the known natural abundances of $\mathrm{Ge}$ isotopes (IUPAC ${ }^{54}$ ) to calculate the contribution of Ge on mass 74 and mass 76 (Eq. 5): 


\section{Hydride corrections}

Hydrides of isotopes of selenium and other impurities still present in samples after column chromatography will all enter the analyser part of the mass spectrometer and interfere on mass $(\mathrm{N}+1)$. Previous studies have shown that germanium can form hydrides in the hydride generator at a similar rate as selenium ${ }^{33,34}$. Hydrides of ${ }^{73} \mathrm{Ge}$ and ${ }^{76} \mathrm{Ge}$ would be created at mass 74 and 77, respectively, and interfere with Se measurements. However, given the low Ge contents of post-Se purification chemistry samples, the contribution of Ge hydrides at mass $74\left({ }^{73} \mathrm{GeH}\right)$ and $77\left({ }^{76} \mathrm{GeH}\right)$ is negligible and, as shown in previous studies, no $\mathrm{GeH}$ correction in purified samples is needed ${ }^{33,34}$. However, the hydrides ${ }^{76} \mathrm{SeH}$ and ${ }^{77} \mathrm{SeH}$ directly interfere on Se isotope masses 77 and 78 and must still be accounted for. The hydride generation rate $\left(\mathrm{HG}_{\text {rate }}\right)$ is estimated by measuring ${ }^{82} \mathrm{SeH}$ on mass 83 (see Table 2) and using the calculated intensity of ${ }^{82} \mathrm{Se}_{\text {corr }}$ (Eq. 3), as defined in the following equation:

$$
H G_{\text {rate }}=\frac{{ }^{82} \mathrm{SeH}}{{ }^{82} \mathrm{Se}_{\mathrm{corr}}}
$$

Eq. 6

Typically, $10^{-4}<\mathrm{HG}_{\text {rate }}<5^{*} 10^{-4}$ and is very stable throughout one mass spectrometer session. The hydride generation rate is assumed to be equal for all selenium isotopes and $\mathrm{HG}_{\text {rate }}$ is used to correct for the contribution of ${ }^{76} \mathrm{SeH}$ and ${ }^{77} \mathrm{SeH}$ on ${ }^{77} \mathrm{Se}$ and ${ }^{78} \mathrm{Se}$ signals respectively (see Fig. 2, green path, hydride correction path):

${ }^{7 X} \mathrm{SeH}={ }^{7 X} \mathrm{Se} * H G_{\text {rate }}$ Eq. 7 with $X=6$ or 7

Argon dimers, germanium and arsenic can also form hydrides that will interfere with Se isotopes. Argon dimers of mass $76\left({ }^{38} \mathrm{Ar}^{38} \mathrm{Ar}\right.$ and $\left.{ }^{40} \mathrm{Ar}^{36} \mathrm{Ar}\right)$ form hydrides that interfere on 
mass 77. The hydride generation rate for argon dimers is different from the selenium hydride $\mathrm{HG}_{\text {rate, as experimentally demonstrated by Pogge von Strandmann et al., 2014 }}{ }^{33}$, which is expected as ArAr is a molecule while Se is an atom. It is therefore not possible to correct for the contribution of ArArH on mass 77 using the hydride generation rate estimated with the Se ion beam. However, the contribution of ArArH can be corrected in a straightforward manner using sample standard bracketing, as the argon dimer generation rate is similar in samples and standards of equal Se concentration (+/- 5\%).

Although ${ }^{75} \mathrm{As}$ does not directly interfere on any Se isotope masses, ${ }^{75} \mathrm{As}$ can form ${ }^{75} \mathrm{AsH}$ hydrides if present in the analyte, thus interfering on ${ }^{76} \mathrm{Se}$. To correct for the arsenic hydride contribution on mass 76 , we assume that the hydride generation rate for ${ }^{75} \mathrm{AsH}$ is similar to that of selenium isotopes (see Fig. 2, green path, hydride correction path). Mass 75 is monitored throughout the measurement to get the ${ }^{75} \mathrm{As}$ signal. The ${ }^{75} \mathrm{AsH}$ contribution on mass 76 is therefore:

$$
{ }^{75} \text { AsH }=\quad{ }^{75} A s_{\text {corr }} * H G_{\text {rate }}={ }^{75} A s * \frac{{ }^{82} S e H}{{ }^{82} S e_{\text {corr }}} \quad \text { Eq. } 8
$$

With ${ }^{75} \mathrm{As}_{\text {corr }}:{ }^{75} \mathrm{As}$ corrected for ${ }^{40} \mathrm{Ar}{ }^{35} \mathrm{Cl}$ using the on peak zero measurement.

\section{Results and discussion}

\section{Internal precision on $\delta^{82 / 78} \mathrm{Se}$ for a single analysis of one block of 80 cycles, 4 seconds} integration time, typically ranges from 0.010 to $0.025 \%$ (95\% c.i., 2 s.e.), as shown in Fig. 3 , which compares well with the predicted internal errors for similar beam intensity. Larger errors reported for some measurements are linked to short-term instability of the argon dimer and/or 
hydride generation rates (see Fig. 3). Once errors on bracketing standards are propagated, internal errors on bracketed sample data typically range 0.014 to $0.033 \%$ o (95\% c.i.). Our estimates of long-term reproducibility are discussed in further detail below.

\subsection{NIST SRM 3149 scaling to Merck Se}

In this study, we report the isotopic composition of the international Se standard reference material NIST SRM 3149 relative to the same MERCK Se solution first measured by Rouxel et al., $2002^{6}$, then calibrated by Carignan and Wen in $2007^{35}$. We measured the MERCK Se solution 93 times during 15 different analytical sessions, spanning from June 2014 to January 2016, from $n=4$ to $n=9$ times at each session. Results are presented in Fig. 4. During individual analytical sessions, the isotopic composition of the Se MERCK solution relative to Se NIST SRM-3149 was between -0.958 and $-1.027 \%$ with a 2 s.d. error ranging from 0.015 $\%$ for the best session $(n=6)$ to $0.030 \%$ for the less reproducible one $(n=7)$. We obtained an overall $\delta^{82 / 78}$ Se composition for the MERCK Se solution of $-0.988 \pm 0.034 \%$ o ( 2 s.d. from the weighed means of analytical sessions, total number of analyses $n=93$ ) relative to the Se NIST SRM-3149 international standard, which is well within analytical precision of Carignan and Wen's calibrated value of $-1.03 \pm 0.20 \%{ }^{35}$. Taking into account all measurements $(\mathrm{n}=$ 93), Se MERCK yields a value of $\delta^{82 / 78} \mathrm{Se}=-0.989 \pm 0.040 \%$ ( 2 s.d., $\left.\mathrm{n}=93\right)$, which is our long-term average standard reproducibility.

Our protocol have allowed us to improve on the reproducibility of Se isotope measurements, as reported in recent published studies, such as in Kurzawa et al., 2017 ( 2 s.d. $=0.13 \%$ on $\delta^{82 / 78} \mathrm{Se}$ for repeated measurements of the MH-495 Se standard, $\mathrm{n}=100^{29}$ ) or in Chang et al., 2017 ( 2 s.d. $=0.07 \%$ on $\delta^{82 / 78}$ Se for repeated measurements of Merck lot HC44698550 and 
349 lot HC44697996 Se standards, $n=24$ and 10 respectively ${ }^{56}$ ). However, direct comparison of these methods is difficult as these different studies involved Se isotope analyses carried out on solutions of different Se concentrations and hence beam intensities. For example, in Kurzawa et al., 2017, measurements were performed at $15 \mathrm{ppb}$ Se solution, while the measurements in our study involved solutions of $50 \mathrm{ppb}$ Se. The effect of counting statistics related to differences in concentration can be calculated, which gives us access to an estimate of our potential reproducibility at $15 \mathrm{ppb}: 0.073 \%$, roughly half that obtained by Kurzawa et al., 2017. As we have accounted for Johnson noise this difference must be related to different hydride and argon dimers correction strategies ${ }^{29}$. As for Chang et al., 2017, it is unclear at what concentration they performed their measurements, therefore no direct comparison should be drawn ${ }^{56}$.

\subsection{Robustness of the method}

\section{Correction of isobaric interferences related to germanium and arsenic}

The accuracy of the Ge corrections used in our protocol were tested by measuring multiple Gedoped selenium solutions. Results are shown in Fig. 5. Solutions with a Ge/Se ratio of up to 0.3 were tested, which represents $\sim 3$ times the maximum post-TCF (thiol cotton fibre) chemistry Ge/Se ratio in detrital sediments ${ }^{33}$. Up to a post-TCF chemistry Ge/Se ratio of 0.3 , our corrected measurements are within the long-term external reproducibility of $0.034 \%$ of un-doped standards (2 s.d.; see Fig. 5). For samples highly enriched in germanium such as some hydrothermal veins and deposits ${ }^{57}$, TCF chemistry might produce Ge/Se ratios greater than 1 , in which case the monitoring of a second Ge mass (e.g. ${ }^{72} \mathrm{Ge}$ ) might prove useful to independently monitor Ge mass bias, rather than assuming that it is identical to that of Se. Another way to reduce the $\mathrm{Ge} / \mathrm{Se}$ ratio in measured samples is to use the anion/cation-exchange resin-based Se purification and extraction protocol recently developed by Kurzawa et al 
373 (Kurzawa et al., 2017, method developed and adapted after Fehr et al., 2004 and Wang et al.,

374 2013), that gives better and more robust Se purification than the TCF protocol ${ }^{29,58,59}$.

The accuracy of the arsenic hydride correction was tested by measuring several As-doped NIST SRM-3149 selenium standard solutions, with As/Se ranging from 0.02 to 0.5. Results are plotted in Fig. 6. For $0<\mathrm{As} / \mathrm{Se}<0.3$, all corrected measurements yield the correct Se NIST SRM -3149 isotopic composition within error with our long-term external reproducibility $( \pm 2$

s.d. $=0.034 \%$ ). Average post-TCF chemistry As/Se ratios are typically $<0.15$ for detrital sediments, as shown by Pogge von Strandmann et al. in their 2014 study $^{33}$. The maximum postTCF chemistry As/Se ratio obtained by by Pogge von Strandmann et al. is $0.3^{33}$, meaning that our arsenic hydride correction is adequate for TCF chemistry-processed samples with similar matrices. Prior to using this correction on Se samples processed via anion/cation exchange resin column chemistry ${ }^{29,58,59}$, further tests on the final As/Se ratio should be performed.

\section{Effect of spike/sample non-ideal mixtures}

The developed ${ }^{76} \mathrm{Se}-{ }^{78} \mathrm{Se}$ double spike is designed to give the best error for a $50 \%$ sample $50 \%$ double spike mixture (see Fig. 1). The double spike fraction, $f_{\text {spike, }}$, is equal to 0.5 for this ideal mixture (see Fig. 7). Achieving the perfect selenium sample to spike ratio to perform Se isotope measurements is not trivial, for two major reasons. The first issue is that it is difficult to precisely measure Se concentrations in geological samples, because (i) terrestrial and extraterrestrial rocks, with the exception of some Se-rich sediments such as shales, contain very low amount of selenium (e.g. from 1 to $100 \mathrm{ppb}$ in peridotites, from 1 to $200 \mathrm{ppb}$ in igneous rocks, 
to those discussed in previous sections - in particular the low Se ionization and the argon $\operatorname{dimers}^{26,27}$.

The other major issue is directly related to the low abundance of selenium in geological samples: precisely measuring one sample's concentration may simply require too much of the sample concerned. This is particularly limiting when dealing with precious and unique samples such as meteorites (e.g. Murchison, $12.2 \mathrm{ppm} \mathrm{Se} \mathrm{S}^{22}$ ) or rare and old fossils. In this context, it is of primary importance to assess the effect of non-ideal spike to sample mixtures on the measurement of Se isotopes and to determine how tolerant our ${ }^{76} \mathrm{Se}-{ }^{78} \mathrm{Se}$ double spike is toward such mixing uncertainties. In order to test this effect, several non-ideal spike to Se NIST SRM3149 (sample) mixtures were measured, with the double spike fraction $f_{\text {spike }}$ ranging from 0.2 (mixture: $20 \%{ }^{76} \mathrm{Se}-{ }^{78} \mathrm{Se}$ double spike $+80 \%$ sample Se NIST SRM-3149) to 0.8 (mixture: $80 \%{ }^{76} \mathrm{Se}-{ }^{78}$ Se double spike $+20 \%$ sample Se NIST SRM-3149). Results are shown in Fig. 7. For $0.3<f_{\text {spike }}<0.7$, the Se NIST SRM-3149 $\delta^{82 / 78}$ Se values are within the long-term external reproducibility of $0.034 \%$ ( 2 s.d.) and indistinguishable from the ideal 50\% double spike, $50 \%$ sample mixture. Outside this $f_{\text {spike }}$ range, the measured values should be discarded, as the offset from the right value increases quickly $\left(0.18 \%\right.$ for $f_{\text {spike }}=0.2 ; 0.71 \%$ for $f_{\text {spike }}=0.8$; see Fig. 7).

\section{Effect of standard/sample concentration mismatch}

417 Our method combines the use of $\mathrm{a}^{76} \mathrm{Se}-{ }^{78} \mathrm{Se}$ double spike to sample-standard bracketing (SSB), by bracketing each sample with two measurements of a double-spiked NIST SRM-3149 Se pure standard. The use of SSB along with double spike allows us to correct for small variations in gas-based interferences and hydride generation (e.g. for bromine hydrides ${ }^{79} \mathrm{BrH}$ and ${ }^{81} \mathrm{BrH}$ on masses 80 and 82 respectively or argon chloride molecules $\mathrm{ArCl}$ on mass 77; see table 2) 
which are not directly accounted for in our interference corrections and hence double-spike deconvolution. As for isotope measurement methods relying purely on SSB (e.g. $\mathrm{Zn}^{61,62}$, Fe and $\mathrm{Cu}^{63}$ ), measured samples and standards should share the same concentration. We have tested the effect of sample/standard concentration mismatch on the quality and reproducibility of our Se isotope measurements (see Fig. 8). Samples with concentration that differs from the standard concentration by systematic amounts were measured and bracketed by a doublespiked NIST SRM-3149 Se pure standard of concentration 125 ppb. Concentration mismatch has a large effect on $\delta^{82 / 78} \mathrm{Se}$ measurements. A concentration mismatch of $+/-10 \%$ can generate an error on the $\delta^{82 / 78}$ Se value of 0.1 to $0.2 \%$ (see Fig. 8), which is considerably greater than our long-term reproducibility $(2$ s.d. $=0.034 \%$ ) calculated on sample/standards of equal concentration. A concentration mismatch greater than $45 \%$ will impact the $\delta^{82 / 78}$ Se value by more than $1 \%$ (Fig. 8). Our tests show that concentration matching between the samples and the standard is the most critical parameter to optimise in these measurements. Sample dilution before measurements on a MC-ICPMS therefore needs to be done extremely carefully, and in case of concentration mismatch larger than $5 \%$, we recommend discarding the data and performing the measurement again.

\section{Conclusions}

This study presents a methodology to precisely measure selenium isotopes, based on the development of a ${ }^{76} \mathrm{Se}^{78} \mathrm{Se}$ Se double-spike. Our double spike was designed to minimise the error on Se isotope measurements, and while our choice of ${ }^{76} \mathrm{Se}$ and ${ }^{78} \mathrm{Se}$ as spiked elements is unusual - as both these isotopes show interferences with argon dimers - we can demonstrate that this issue can be addressed with systematic interference corrections made prior to spike deconvolution. In particular, the monitoring of argide formation on mass 80 and the subtraction of argon dimers contribution on mass 76 and 78 at each integration, during the measurement, 
has proven essential. Our setting for the hydride generation coupled to a ThermoFisher ${ }^{\mathrm{TM}}$ Scientific Neptune Plus ${ }^{\mathrm{TM}}$ MC-ICPMS produces an overall sensitivity over $1000 \mathrm{~V}$ per ppm of Se. By combining this improved sensitivity with our double spike and an integration-by450 integration argon dimer correction, our method requires $25 \mathrm{ng}$ of natural Se to perform one

451 measurement, and we obtain a long-term external reproducibility of $0.040 \%$ ( 2 s.d., $n=93)$. This method should enable measurement of the Se isotope composition of a wide array of geological samples, thus firmly establishing Se stable isotopes as a novel addition to the geochemist toolbox.

\section{Conflicts of interest}

There are no conflicts of interest to declare.

\section{Acknowledgments}

This work was supported by ThermoScientific, an ERC Starting Grant (HabitablePlanet; 306655), and two NERC Consortium Grants (“Deep Volatiles”, NE/M0003/1 and "Tellurium and Selenium Cycling and Supply (TeASe)" NE/M010848/1) awarded to HW. HW and MLP also acknowledge salary support from a NERC Advanced Fellowship (NE/F014295/2) and a MSCA Individual Fellowship (SPECADIS, 794825), respectively. We thank Philip Pogge von Strandmann for helpful discussions and tips to set up the measurements, Kathrin Schilling and Tom Johnson for their help and advice at throughout the project. 
1 A. Galy, N. S. Belshaw, L. Halicz and R. K. O’Nions, Int. J. Mass Spectrom., 2001, 208, 89-98.

2 M.-A. Millet and N. Dauphas, J. Anal. At. Spectrom., 2014, 29, 1444.

3 C. Siebert, T. F. Nägler and J. D. Kramers, Geochem. Geophys. Geosystems, 2001, 2, n/a-n/a. 306.

5 N. Dauphas and O. Rouxel, Mass Spectrom. Rev., 2006, 25, 515-550.

6 O. Rouxel, J. Ludden, J. Carignan, L. Marin and Y. Fouquet, Geochim. Cosmochim. Acta, 2002, 66, 3191-3199.

7 M. A. Kipp, E. E. Stüeken, A. Bekker and R. Buick, Proc. Natl. Acad. Sci., 2017, 114, 875-880.

8 E. E. Stüeken, R. Buick and A. D. Anbar, Geology, 2015, 43, 259-262.

9 H. Wen and J. Carignan, Geochim. Cosmochim. Acta, 2011, 75, 1411-1427.

10 H. Wen, J. Carignan, X. Chu, H. Fan, C. Cloquet, J. Huang, Y. Zhang and H. Chang, Chem. Geol., 2014, 390, 164-172.

11 E. E. Stüeken, R. Buick, A. Bekker, D. Catling, J. Foriel, B. M. Guy, L. C. Kah, H. G. Machel, I. P. Montañez and S. W. Poulton, Geochim. Cosmochim. Acta, 2015, 162, 109-125.

12 E. E. Stüeken, Rev. Mineral. Geochem., 2017, 82, 657-682.

13 S. König, B. Eickmann, T. Zack, A. Yierpan, M. Wille, H. Taubald and R. Schoenberg, Geochim. Cosmochim. Acta, 2019, 244, 24-39.

14 P. A. E. Pogge von Strandmann, E. E. Stüeken, T. Elliott, S. W. Poulton, C. M. Dehler, D. E. Canfield and D. C. Catling, Nat. Commun., 2015, 6, 10157.

15 K. Schilling, T. M. Johnson and W. Wilcke, Chem. Geol., 2013, 352, 101-107.

16 K. Schilling, T. M. Johnson, K. S. Dhillon and P. R. D. Mason, Environ. Sci. Technol., 2015, 49, 96909698.

17 A. Basu, K. Schilling, S. T. Brown, T. M. Johnson, J. N. Christensen, M. Hartmann, P. W. Reimus, J.

M. Heikoop, G. Woldegabriel and D. J. DePaolo, Environ. Sci. Technol., 2016, 50, 10833-10842.

18 K. Schilling, T. M. Johnson and W. Wilcke, Soil Sci. Soc. Am. J., 2011, 75, 1354.

19 T. M. Johnson, M. J. Herbel, T. D. Bullen and P. T. Zawislanski, Geochim. Cosmochim. Acta, 1999, 63, 2775-2783.

20 M. J. Herbel, T. M. Johnson, R. S. Oremland and T. D. Bullen, Geochim. Cosmochim. Acta, 2000, 64, 3701-3709.

21 T. Kurzawa, S. König, J. C. Alt, A. Yierpan and R. Schoenberg, Chem. Geol., 2019, 513, 239-249. 22 J. Labidi, S. König, T. Kurzawa, A. Yierpan and R. Schoenberg, Earth Planet. Sci. Lett., 2018, 481, 212-222.

23 H. Vollstaedt, K. Mezger and I. Leya, Earth Planet. Sci. Lett., 2016, 450, 372-380.

24 A. Yierpan, S. König, J. Labidi and R. Schoenberg, Geochim. Cosmochim. Acta, 2019, 249, 199-224.

25 M. I. Varas-Reus, S. König, A. Yierpan, J.-P. Lorand and R. Schoenberg, Nat. Geosci., 2019, 12, 779 782.

26 J.-P. Lorand and O. Alard, Chem. Geol., 2010, 278, 120-130.

27 S. König, A. Luguet, J.-P. Lorand, F. Wombacher and M. Lissner, Geochim. Cosmochim. Acta, 2012, 86, 354-366.

28 J.-P. Lorand, O. Alard, A. Luguet and R. R. Keays, Geochim. Cosmochim. Acta, 2003, 67, 41374151.

29 T. Kurzawa, S. König, J. Labidi, A. Yierpan and R. Schoenberg, Chem. Geol., 2017, 466, 219-228.

30 K. Schilling, T. M. Johnson and P. R. D. Mason, Chem. Geol., 2014, 381, 125-130.

31 A. Yierpan, S. König, J. Labidi, T. Kurzawa, M. G. Babechuk and R. Schoenberg, Geochem. Geophys. Geosystems, 2018, 19, 516-533.

32 H. Vollstaedt, K. Mezger, T. Nägler, I. Leya and A. Trinquier, Int. J. Mass Spectrom., 2016, 401, 5563. 
33 P. A. E. Pogge von Strandmann, C. D. Coath, D. C. Catling, S. W. Poulton and T. Elliott, J Anal Spectrom, 2014, 29, 1648-1659.

34 E. E. Stüeken, J. Foriel, B. K. Nelson, R. Buick and D. C. Catling, J. Anal. At. Spectrom., 2013, 28, 1734.

35 J. Carignan and H. Wen, Chem. Geol., 2007, 242, 347-350.

36 F. Albarede and B. Beard, Rev. Mineral. Geochem., 2004, 55, 113-152.

37 B. Hamelin, G. Manhes, F. Albarede and C. J. Allègre, Geochim. Cosmochim. Acta, 1985, 49, 173182.

38 M. H. Dodson, J. Sci. Instrum., 1963, 40, 289-295.

39 M. H. Dodson, Geochim. Cosmochim. Acta, 1970, 34, 1241-1244.

40 J. F. Rudge, B. C. Reynolds and B. Bourdon, Chem. Geol., 2009, 265, 420-431.

41 M. H. Dodson, J. Phys. [E], 1969, 2, 490-498.

42 S. G. John, J. Anal. At. Spectrom., 2012, 27, 2123.

43 F. Kurzweil, M. Wille, N. Gantert, N. J. Beukes and R. Schoenberg, Earth Planet. Sci. Lett., 2016, 452, 69-78.

44 G. Albut, M. G. Babechuk, I. C. Kleinhanns, M. Benger, N. J. Beukes, B. Steinhilber, A. J. B. Smith, S.

J. Kruger and R. Schoenberg, Geochim. Cosmochim. Acta, 2018, 228, 157-189.

45 M.-A. Millet, J. A. Baker and C. E. Payne, Chem. Geol., 2012, 304-305, 18-25.

46 A. J. McCoy-West, M.-A. Millet and K. W. Burton, Earth Planet. Sci. Lett., 2017, 480, 121-132.

47 J. A. M. Nanne, M.-A. Millet, K. W. Burton, C. W. Dale, G. M. Nowell and H. M. Williams, J. Anal. At. Spectrom., 2017, 32, 749-765.

48 S. J. G. Galer, Chem. Geol., 1999, 157, 255-274.

49 T. Kurzawa, S. König, J. Labidi, A. Yierpan and R. Schoenberg, Chem. Geol., 2017, 466, 219-228. 50 J.-M. Zhu, T. M. Johnson, S. K. Clark, X.-K. Zhu and X.-L. Wang, Geochim. Cosmochim. Acta, 2014, 126, 228-249.

51 J. Wang, T. Ren, H. Lu, T. Zhou and M. Zhao, Int. J. Mass Spectrom., 2011, 308, 65-70.

52 S. K. Clark and T. M. Johnson, Environ. Sci. Technol., 2008, 42, 7850-7855.

53 N. Elwaer and H. Hintelmann, J. Anal. At. Spectrom., 2008, 23, 733.

54 Meija Juris, Coplen Tyler B., Berglund Michael, Brand Willi A., De Bièvre Paul, Gröning Manfred, Holden Norman E., Irrgeher Johanna, Loss Robert D., Walczyk Thomas and Prohaska Thomas, Pure Appl. Chem., 2016, 88, 293.

55 J.-Y. Lee, K. Marti, J. P. Severinghaus, K. Kawamura, H.-S. Yoo, J. B. Lee and J. S. Kim, Geochim. Cosmochim. Acta, 2006, 70, 4507-4512.

56 Y. Chang, J. Zhang, J.-Q. Qu and Y. Xue, Chem. Geol., 2017, 471, 65-73.

57 F. Sahlström, A. Arribas, P. Dirks, I. Corral and Z. Chang, Minerals, 2017, 7, 213.

58 M. A. Fehr, M. Rehkämper and A. N. Halliday, Int. J. Mass Spectrom., 2004, 232, 83-94.

59 Z. Wang and H. Becker, Nature, 2013, 499, 328.

60 Y. Tamari, H. Ogawa, Y. Fukumoto, H. Tsuji and Y. Kusaka, Bull. Chem. Soc. Jpn., 1990, 63, 26312638.

61 M.-L. Pons, B. Debret, P. Bouilhol, A. Delacour and H. Williams, Nat. Commun., 2016, 7, 13794.

62 C. N. Maréchal, P. Télouk and F. Albarède, Chem. Geol., 1999, 156, 251-273.

63 K. Jaouen, M.-L. Pons and V. Balter, Earth Planet. Sci. Lett., 2013, 374, 164-172. 


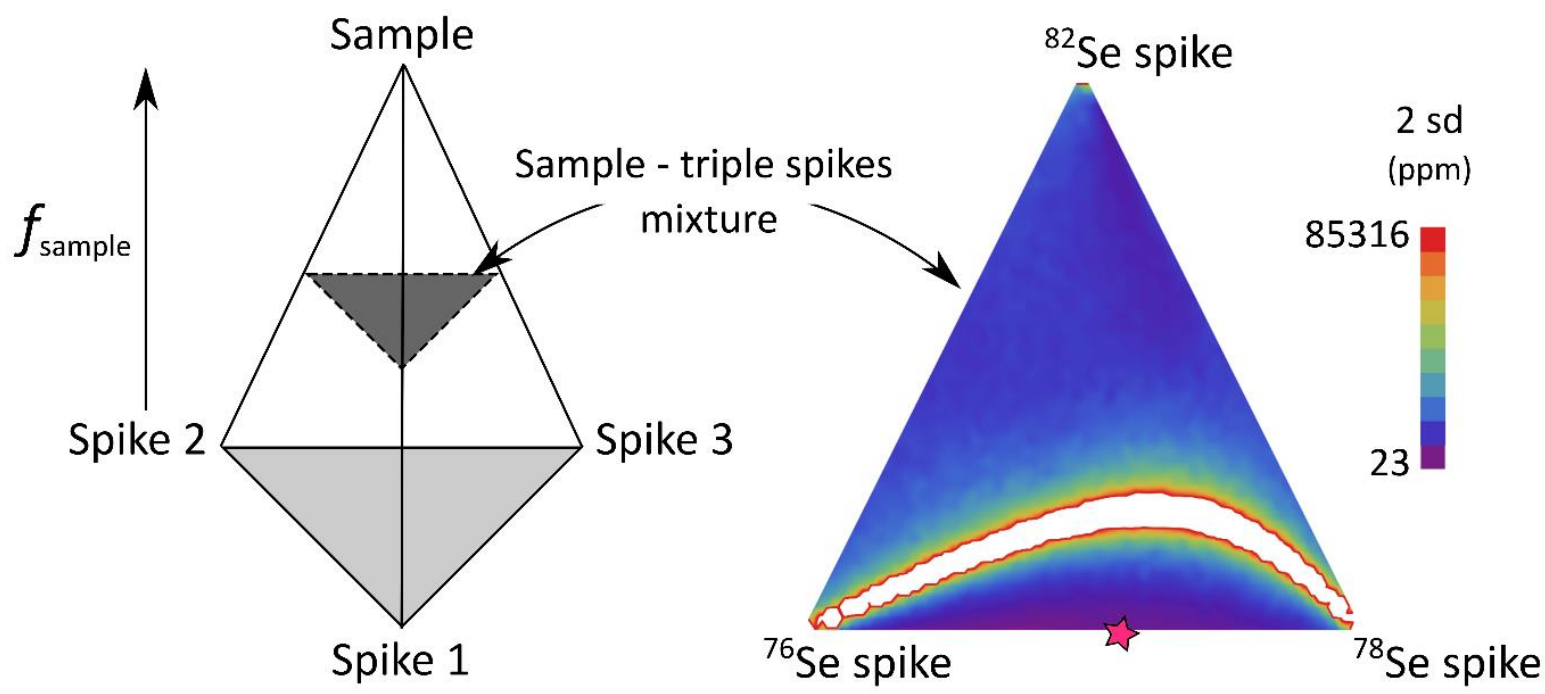

Fig. 1: Schematic representation of all the possible spike-sample mixtures investigated in our 575 triple spike Monte-Carlo simulation. All compositions are enclosed in a tetrahedron which top 576 apex is the standard composition (i.e., natural stable isotope composition) and base apexes 577 (light grey area) are the individual spikes. In this tetrahedron, sections parallel to the base 578 (represented in dark grey) contain all possible triple spike mixtures mixed with the same 579 amount of natural sample. 


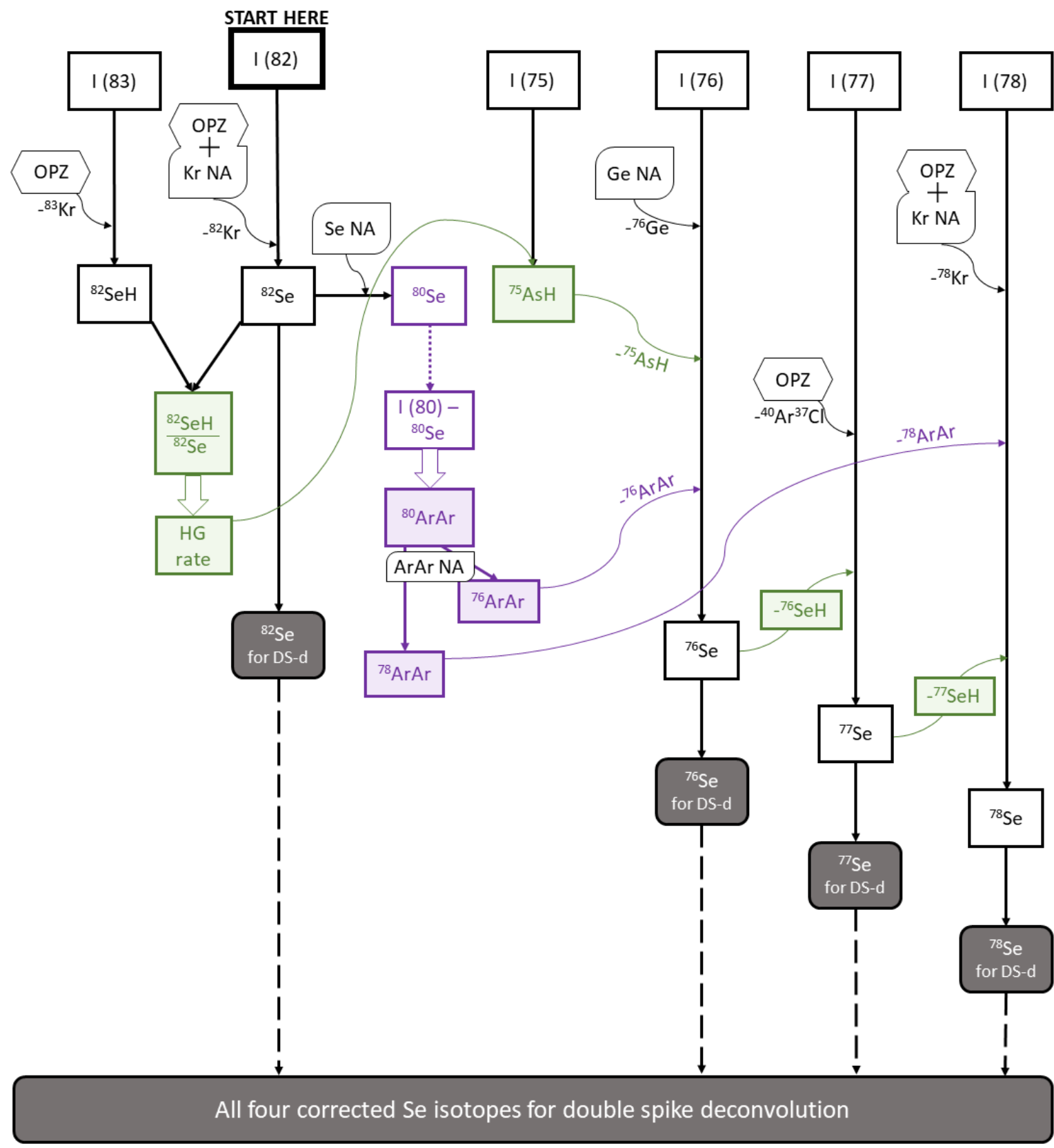

581

Fig. 2: Flowchart illustrating the corrections done to the intensities on masses 76, 77, 78 and 82 to extract the real selenium signal from all the isobaric interferences prior to double spike deconvolution, as described in paragraph 4. OPZ: on peak zero. NA: natural abundances. HG rate: hydride generation rate. DS-d: double spike deconvolution. In green: hydride correction. In purple: argon dimer correction. 


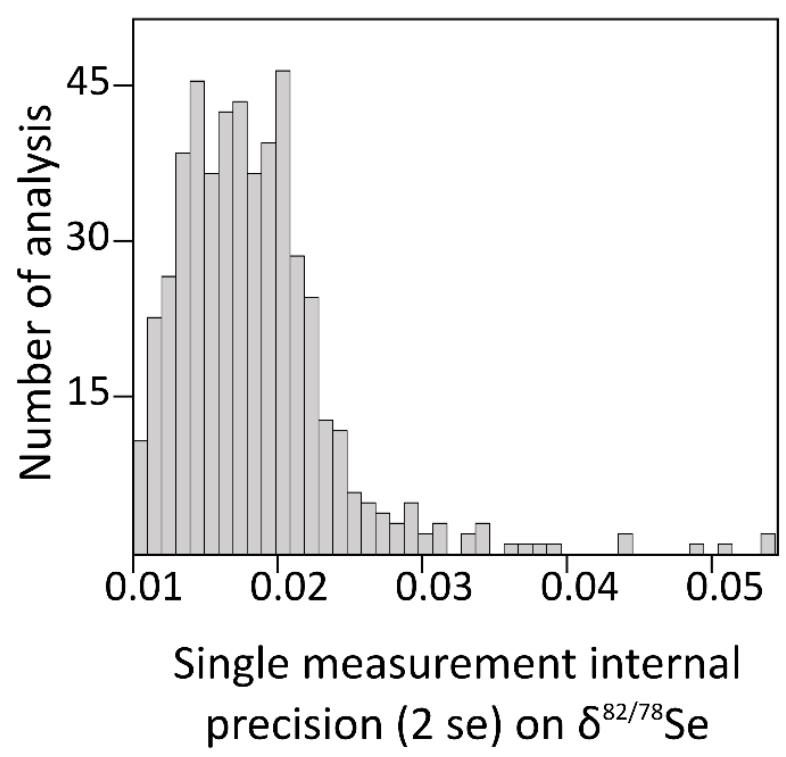

591

592 Fig. 3: Internal precision on $\delta^{82 / 78}$ Se for all single measurements included in this study (2 s.e.), 593 for 1 block of 80 cycles, 4 seconds integration time. Errors typically range from ca. 0.01 to $5940.025 \%$ (95\% c.i.) although some larger errors can occur when the mass spectrometer is 595 unstable. Once errors on bracketing standards are taken into account, errors on sample data 596 typically range 0.014 to $0.033 \%$ (95\% c.i.).

597

598

599 


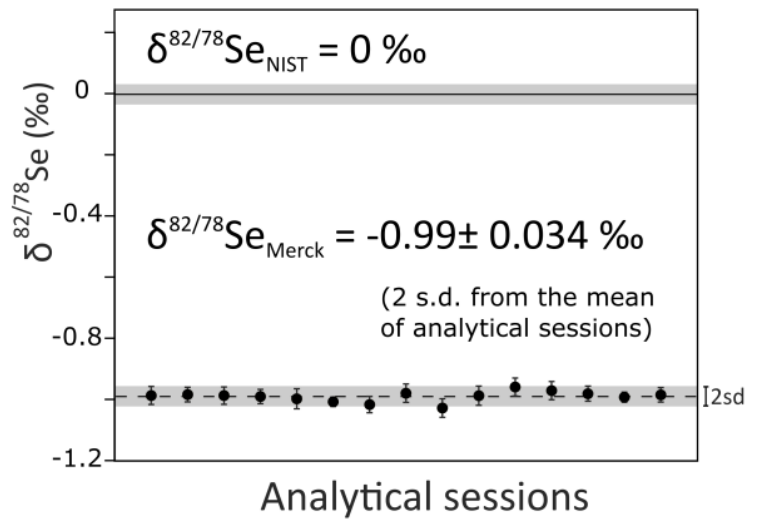

602

603 Fig. 4: Merck Se solution calibration to Se NIST SRM-3149. Results obtained during all the analytical sessions. Error bars are 2 times the standard deviation for results obtained during individual sessions ( $\mathrm{n}=4$ to 9 ). The grey area corresponds to the long-term external reproducibility ( \pm 2 s.d. $=0.040 \%, \mathrm{n}=93$ ). The value Merck Se relative to Se NIST SRM 3149 is: $\delta^{82 / 78} \mathrm{Se}_{\text {Merck }}=-0.99 \pm 0.034 \%$ ( 2 s.d., from the mean of analytical sessions), which is in perfect agreement with previous calibration by Carignan and Wen, $2007\left(\delta^{82 / 78} \mathrm{Se}_{\text {Merck }}=-\right.$ $1.03 \pm 0.20 \%$, Carignan and Wen, 2007). 


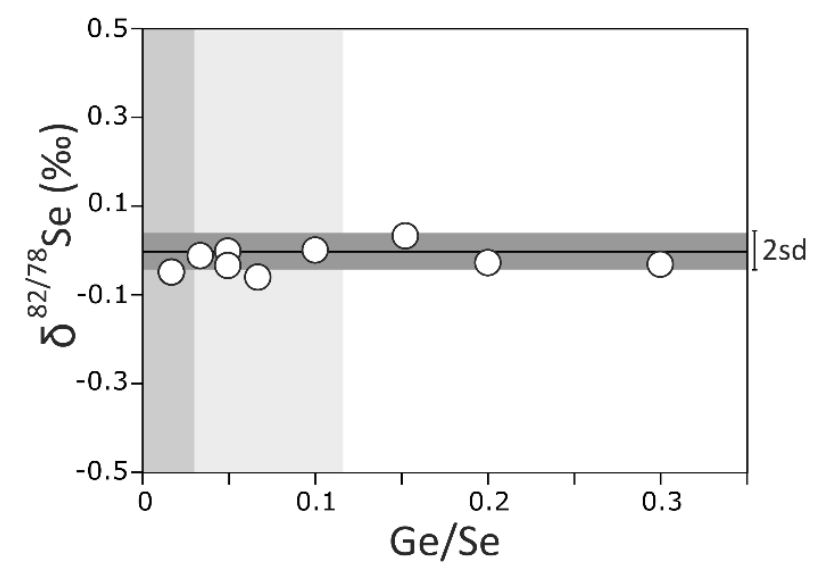

614

615 Fig. 5: Ge doping experiments on NIST SRM -3149 and effect on the Se isotope composition 616 measurement. The solid black line represents $0 \%$ and the dark grey area corresponds to the 617 long-term external reproducibility $( \pm 2$ s.d. $=0.040 \%$ o $)$. On a single analysis $(n=80)$, the 618 internal error for $\delta^{82 / 78} \mathrm{Se}$ is $0.010<2$ s.e. $<0.025 \%$, which is smaller than the marker's size. 619 The light grey area represents the average post-TCF chemistry Ge/Se ratio, and the medium 620 grey area represents the maximum post-TCF chemistry Ge/Se ratio (Pogge von Strandmann et 621 al., 2014). 


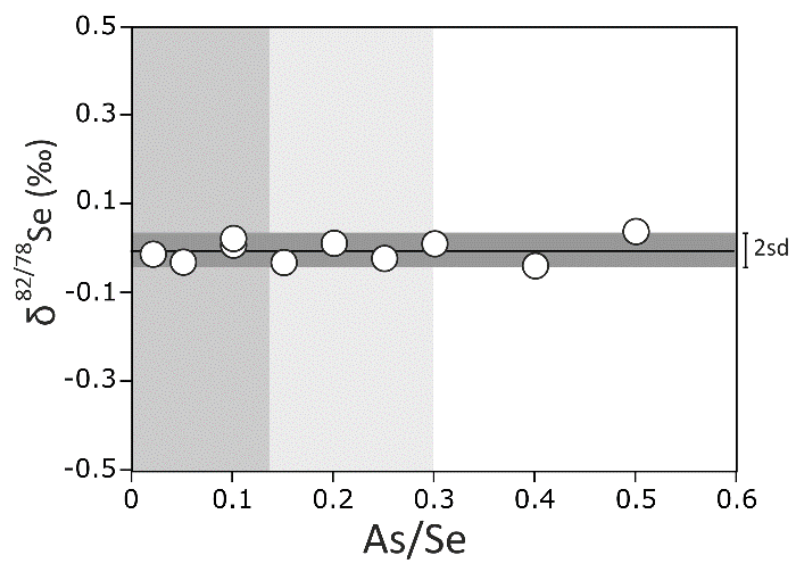

628 Fig. 6: As doping experiments on NIST SRM -3149 and effect on the Se isotope composition 629 measurement. The solid black line represents $0 \%$ and the dark grey area corresponds to the 630 long-term external reproducibility $( \pm 2$ s.d. $=0.040 \%)$. On a single analysis $(n=80)$, the 631 internal error for $\delta^{82 / 78} \mathrm{Se}$ is $0.010<2$ s.e. $<0.025 \%$, which is smaller than the marker's size. 632 The light grey area represents the average post-TCF chemistry As/Se ratio, and the medium grey area represents the maximum post-TCF chemistry As/Se ratio (Pogge von Strandmann et al., 2014). 


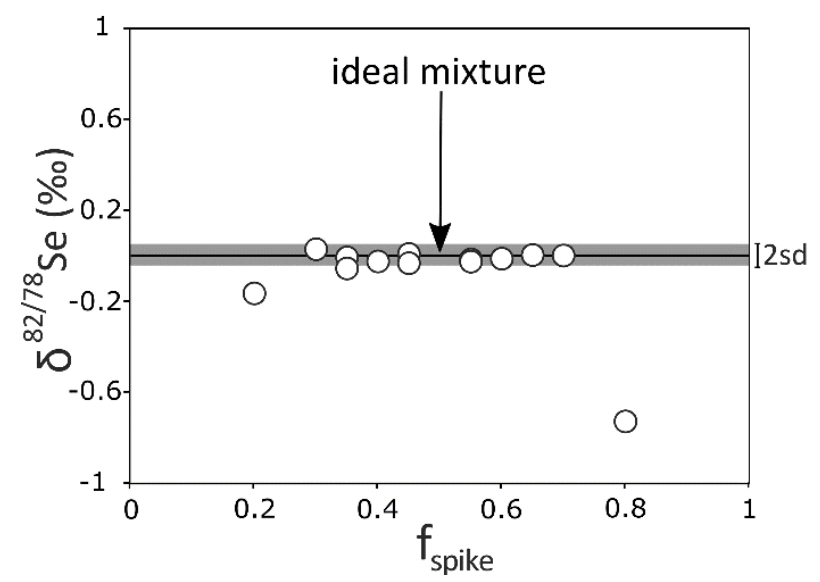

638

639 Fig. 7: Effect of variably spiking Se NIST SRM-3149 on Se isotope measurements using a ${ }^{76} \mathrm{Se}-{ }^{78} \mathrm{Se}$ double spike. The ideal mixture is $50 \%$ sample, $50 \%$ spike $\left(f_{\text {spike }}=0.5\right)$. The solid black line represents $0 \%$ and the dark grey area corresponds to the long-term external reproducibility $( \pm 2$ s.d. $=0.040 \%)$. On a single analysis $(n=80)$, the internal error for $\delta^{82 / 78} \mathrm{Se}$ is $0.010<2$ s.e. $<0.025 \%$, which is smaller than the marker's size. 

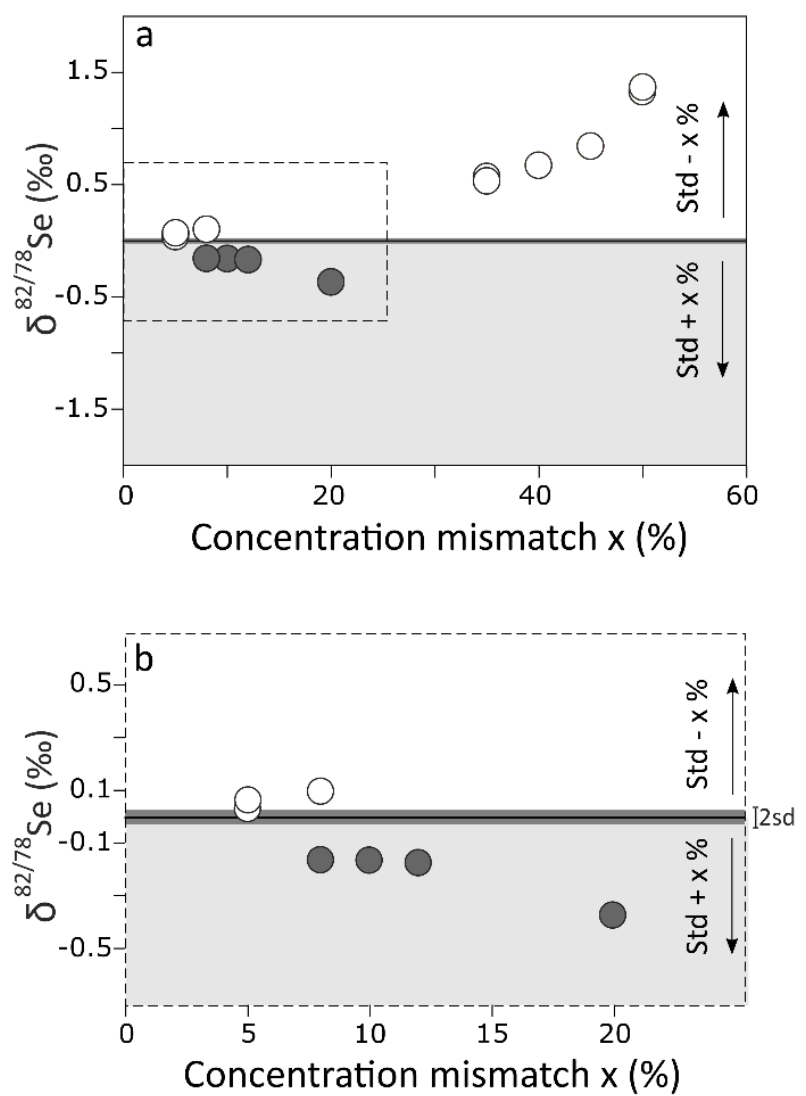

650

651

652 Fig. 8: Effect of sample to standard concentration mismatch on Se isotope measurements. a. 653 Effect of sample-standard concentration mismatch during SSB measurements on the sample $654 \delta^{82 / 78}$ Se. b. Magnification of the dashed area from Fig. a. In both a and b, the solid black line 655 represents $0 \%$ and the dark grey area corresponds to the long-term external reproducibility $( \pm$ 6562 s.d. $=0.040 \%$ ). On a single analysis $(n=80)$, the internal error for $\delta^{82 / 78}$ Se is $0.010<2$ s.e. $657<0.025 \%$, which is smaller than the marker's size. White area and white markers: the sample 658 has a lower concentration than the standard. Light grey area and grey markers: the sample has 659 a greater concentration than the standard. 


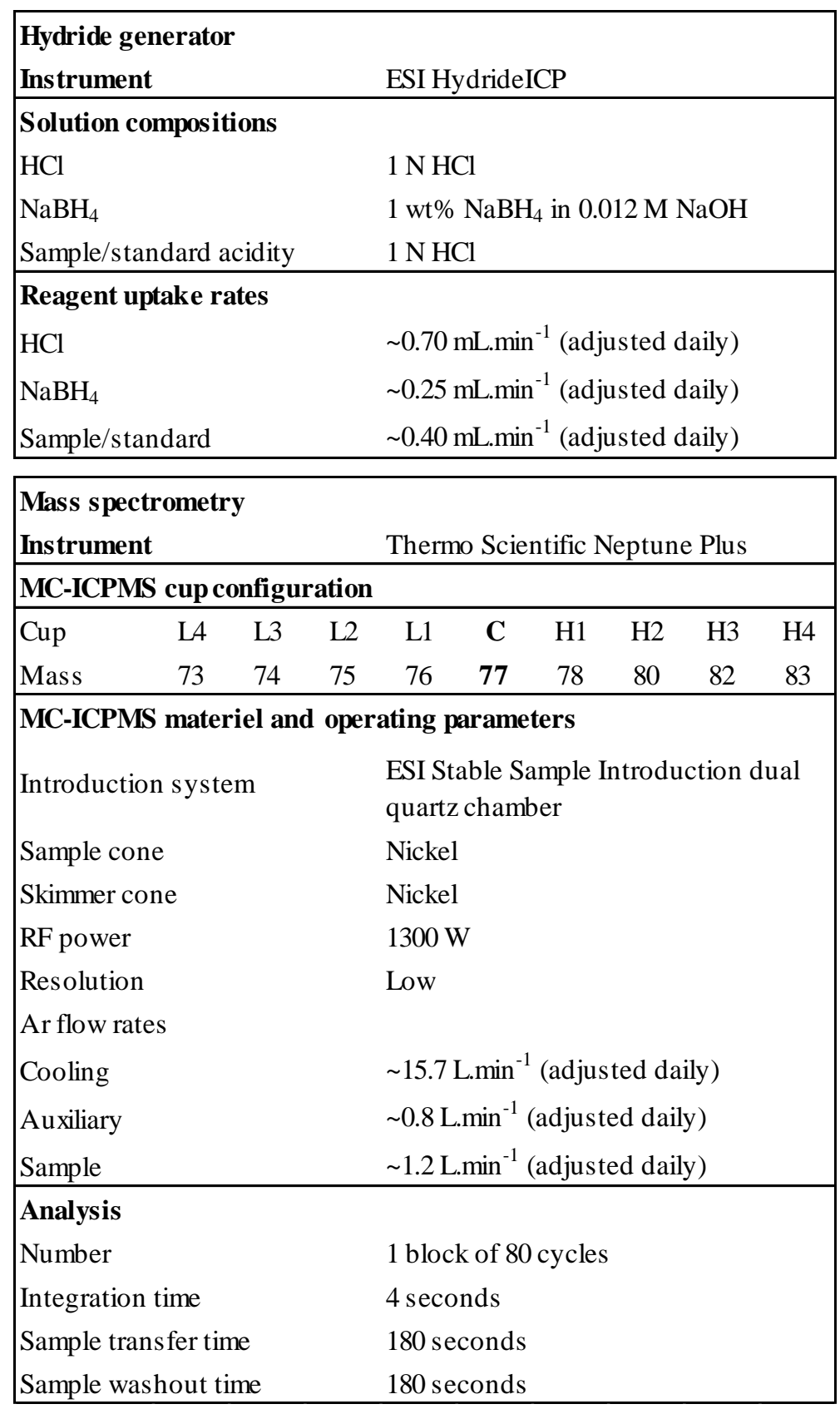




\begin{tabular}{|c|c|c|c|c|}
\hline Cup & $\begin{array}{c}\text { Measured } \\
\text { mass }\end{array}$ & Se isotopes & $\begin{array}{l}\text { Other species and } \\
\text { interferences }\end{array}$ & Correction method \\
\hline L4 & 73 & & ${ }^{73} \mathrm{Ge}$ & used to correct ${ }^{74,76} \mathrm{Ge}$ \\
\hline L3 & 74 & ${ }^{74} \mathrm{Se}$ & ${ }^{74} \mathrm{Ge}$ & manual, using ${ }^{73} \mathrm{Ge}$ \\
\hline $\mathrm{L} 2$ & 75 & & ${ }^{75} \mathrm{As} ;{ }^{75}(\mathrm{SeH}) ;{ }^{75}(\mathrm{ArCl})$ & used to correct $^{76}(\mathrm{AsH})$ \\
\hline $\mathrm{L} 1$ & 76 & ${ }^{76} \mathrm{Se}$ & ${ }^{76}(\mathrm{ArAr}) ;{ }^{76} \mathrm{Ge} ;{ }^{76}(\mathrm{AsH})$ & manual, using: ${ }^{80}(\mathrm{ArAr}) ;{ }^{73} \mathrm{Ge} ;{ }^{75} \mathrm{As}+\mathrm{HG}$ rate \\
\hline $\mathrm{C}$ & 77 & ${ }^{77} \mathrm{Se}$ & $\begin{array}{l}{ }^{77}(\mathrm{SeH}) ;{ }^{77}(\mathrm{ArArH}) ; \\
{ }^{77}(\mathrm{ArCl})\end{array}$ & manual, using: ${ }^{76} \mathrm{Se}+\mathrm{HG}$ rate; and $\mathrm{SSB}$ \\
\hline $\mathrm{H} 1$ & 78 & ${ }^{78} \mathrm{Se}$ & ${ }^{78}(\mathrm{ArAr}) ;{ }^{78}(\mathrm{SeH}) ;{ }^{78} \mathrm{Kr}$ & $\begin{array}{l}\text { manual, using: }{ }^{80}(\mathrm{ArAr}) ;{ }^{: 7} \mathrm{Se}+\mathrm{HG} \text { rate; OPZ } \\
\text { on mass } 83-{ }^{83} \mathrm{Kr}\end{array}$ \\
\hline $\mathrm{H} 2$ & 80 & ${ }^{80} \mathrm{Se}$ & ${ }^{80}(\mathrm{ArAr}) ;{ }^{80} \mathrm{Kr} ;{ }^{80}(\mathrm{BrH})$ & $\begin{array}{l}\text { used to correct Ar dimers; manual, using: OPZ } \\
\text { on mass } 83-{ }^{83} \mathrm{Kr} \text {; SSB }\end{array}$ \\
\hline $\mathrm{H} 3$ & 82 & 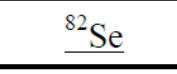 & ${ }^{82} \mathrm{Kr} ;{ }^{82}(\mathrm{BrH})$ & manual, using: OPZ on mass $83-{ }^{83} \mathrm{Kr}$; SSB \\
\hline $\mathrm{H} 4$ & 83 & & ${ }^{83}(\mathrm{SeH}) ;{ }^{83} \mathrm{Kr}$ & $\begin{array}{l}\text { used to assess HG rate; used to correct } \\
{ }^{78,80,82} \mathrm{Kr} \text { during OPZ }\end{array}$ \\
\hline
\end{tabular}

671

Table 2 : Interferences and correction method for Se isotope composition measurement. See main text for a detailed explanation of the corrections used. OPZ = On Peak Zero; HG = Hydride Generation; SSB = Sample Standard Bracketing

675 
Precise measurement of selenium isotopes by HG-MC-ICPMS using a 76-78 double-spike

Marie-Laure Pons ${ }^{\mathrm{a}, \mathrm{b}^{*}}$, Marc-Alban Millet ${ }^{\mathrm{c}}$, Geoff N. Nowell ${ }^{\mathrm{d}}$, Sambuddha Misra ${ }^{\mathrm{a}, \mathrm{e}}$ and Helen M. Williams $^{\mathrm{a}}$

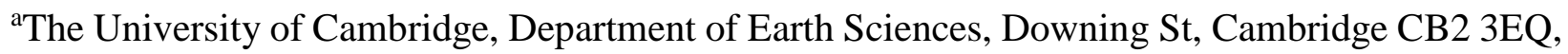
United Kingdom

${ }^{\mathrm{b}} \mathrm{CNRS}$, Aix Marseille Univ, IRD, INRA, Coll France, CEREGE, 13545, Aix en Provence, France ${ }^{\mathrm{c}}$ Cardiff University, School of Earth and Ocean Sciences, Main Building, Park Pl, Cardiff CF10 3AT, United Kingdom

${ }^{\mathrm{d}}$ Durham University, Department of Earth Sciences, Elvet Hill, Durham DH1 3LE, United Kingdom

eIndian Institute of Science, Centre for Earth Sciences, Bengaluru, India

*corresponding author dr.marie.laure.pons@gmail.com

\section{Supplementary information}


Table S1. $\delta^{82 / 78}$ SeMERCK. Merck Se solution calibration to Se NIST SRM-3149. Results obtained during all the analytical sessions.

\begin{tabular}{|c|c|c|c|c|}
\hline $\begin{array}{c}\text { Analytical session } \\
\#\end{array}$ & $\begin{array}{c}\delta^{82 / 78} \operatorname{Se}_{\text {MERCK }} \text { average of } \\
\text { analytical session }\end{array}$ & $\mathrm{n}$ & 2 s.d. & $\delta^{82 / 78} \mathrm{Se}_{\text {MERCK }}$ of single analyses \\
\hline \multirow[t]{7}{*}{1} & -0.9856 & 6 & 0.0293 & \\
\hline & & & & -0.9877 \\
\hline & & & & -0.9581 \\
\hline & & & & -0.9837 \\
\hline & & & & -0.9881 \\
\hline & & & & -0.9981 \\
\hline & & & & -0.9977 \\
\hline \multirow[t]{6}{*}{2} & -0.9827 & 5 & 0.0240 & \\
\hline & & & & -0.9739 \\
\hline & & & & -0.9839 \\
\hline & & & & -0.9939 \\
\hline & & & & -0.9944 \\
\hline & & & & -0.9675 \\
\hline \multirow[t]{8}{*}{3} & -0.9860 & 7 & 0.0285 & \\
\hline & & & & -0.9842 \\
\hline & & & & -0.9666 \\
\hline & & & & -0.9953 \\
\hline & & & & -0.9853 \\
\hline & & & & -1.0034 \\
\hline & & & & -0.9686 \\
\hline & & & & -0.9982 \\
\hline \multirow[t]{4}{*}{4} & -0.9891 & 4 & 0.0232 & -0.9975 \\
\hline & & & & -1.0005 \\
\hline & & & & -0.9797 \\
\hline & & & & -0.9784 \\
\hline \multirow[t]{5}{*}{5} & -0.9964 & 5 & 0.0328 & -1.0008 \\
\hline & & & & -0.9887 \\
\hline & & & & -0.9763 \\
\hline & & & & -1.0207 \\
\hline & & & & -0.9956 \\
\hline
\end{tabular}




\begin{tabular}{|c|c|c|c|c|}
\hline $\begin{array}{c}\text { Analytical session } \\
\#\end{array}$ & $\begin{array}{c}\delta^{82 / 78} \mathrm{Se}_{\mathrm{MERCK}} \text { average of } \\
\text { analytical session }\end{array}$ & $n$ & 2 s.d. & $\delta^{82 / 78} \mathrm{Se}_{\mathrm{MERCK}}$ of single analyses \\
\hline \multirow[t]{7}{*}{6} & \multirow{2}{*}{\multicolumn{4}{|c|}{-1.0072}} \\
\hline & & & & \\
\hline & \multirow[t]{5}{*}{-1.0060} & \multirow[t]{5}{*}{6} & \multirow[t]{5}{*}{3} & -1.0132 \\
\hline & & & & -1.0015 \\
\hline & & & & -1.0002 \\
\hline & & & & -1.0165 \\
\hline & & & & -0.9972 \\
\hline & \multicolumn{4}{|c|}{0.026} \\
\hline \multirow[t]{6}{*}{7} & \multirow[t]{5}{*}{-1.0155} & \multirow[t]{5}{*}{5} & \multirow[t]{5}{*}{6} & -1.0323 \\
\hline & & & & -1.0178 \\
\hline & & & & -1.0168 \\
\hline & & & & -1.0158 \\
\hline & & & & -0.9950 \\
\hline & \multirow{2}{*}{\multicolumn{4}{|c|}{$\begin{array}{r}0.030 \\
4\end{array}$}} \\
\hline \multirow[t]{9}{*}{8} & & & & \\
\hline & & & & -0.9779 \\
\hline & & & & -0.9947 \\
\hline & & & & -0.9648 \\
\hline & & & & -0.9830 \\
\hline & & & & -0.9993 \\
\hline & & & & -0.9613 \\
\hline & & & & -0.9650 \\
\hline & \multicolumn{4}{|c|}{0.029} \\
\hline \multirow[t]{6}{*}{9} & -1.0268 & 5 & 8 & \\
\hline & & & & -1.0384 \\
\hline & & & & -1.0270 \\
\hline & & & & -1.0219 \\
\hline & & & & -1.0045 \\
\hline & & & & -1.0421 \\
\hline \multirow{6}{*}{10} & \multicolumn{4}{|c|}{0.030} \\
\hline & -0.9865 & 4 & 7 & \\
\hline & & & & -0.9790 \\
\hline & & & & -0.9697 \\
\hline & & & & -0.9925 \\
\hline & & & & -1.0047 \\
\hline
\end{tabular}




\begin{tabular}{|c|c|c|c|c|}
\hline $\begin{array}{c}\text { Analytical session } \\
\#\end{array}$ & $\begin{array}{c}\delta^{82 / 78} \mathrm{Se}_{\text {MERCK }} \text { average of } \\
\text { analytical session }\end{array}$ & $\mathrm{n}$ & 2 s.d. & $\delta^{82 / 78} \mathrm{Se}_{\text {MERCK }}$ of single analyses \\
\hline \multirow[t]{5}{*}{11} & -0.9578 & 5 & 0.0292 & -0.9537 \\
\hline & & & & -0.9683 \\
\hline & & & & -0.9773 \\
\hline & & & & -0.9466 \\
\hline & & & & -0.9430 \\
\hline \multirow[t]{13}{*}{12} & -0.9697 & 9 & 0.0296 & \\
\hline & & & & -0.9644 \\
\hline & & & & -0.9691 \\
\hline & & & & -0.9645 \\
\hline & & & & -0.9644 \\
\hline & & & & -0.9871 \\
\hline & & & & -0.9682 \\
\hline & & & & -0.9532 \\
\hline & & & & -0.9644 \\
\hline & & & & -0.9937 \\
\hline & & & & -0.9644 \\
\hline & & & & -0.9947 \\
\hline & & & & -0.9475 \\
\hline \multirow[t]{4}{*}{13} & -0.9799 & 3 & 0.0247 & \\
\hline & & & & -0.9741 \\
\hline & & & & -0.9941 \\
\hline & & & & -0.9715 \\
\hline \multirow[t]{5}{*}{14} & -0.9911 & 4 & 0.0162 & \\
\hline & & & & -1.0015 \\
\hline & & & & -0.9910 \\
\hline & & & & -0.9903 \\
\hline & & & & -0.9817 \\
\hline \multirow[t]{8}{*}{15} & -0.9836 & 9 & 0.0236 & \\
\hline & & & & -0.9882 \\
\hline & & & & -0.9812 \\
\hline & & & & -0.9992 \\
\hline & & & & -0.9701 \\
\hline & & & & -0.9812 \\
\hline & & & & -1.0046 \\
\hline & & & & -0.9718 \\
\hline
\end{tabular}


$-0.9890$

$-0.9670$

$-0.9724$

$-0.9881$

$-0.9902$ 